\title{
Anabases
}

ANABASES Traditions et réceptions de l'Antiquité

14 | 2011

Varia

\section{David BOUVIER et Danielle VAN MAL-MAEDER (éd.), Tradition classique : dialogues avec l'Antiquité}

\section{Geneviève Hoffmann}

\section{OpenEdition}

\section{Journals}

Édition électronique

URL : http://journals.openedition.org/anabases/2357

DOI : 10.4000/anabases.2357

ISSN : 2256-9421

\section{Éditeur}

E.R.A.S.M.E.

\section{Édition imprimée}

Date de publication : 1 octobre 2011

Pagination : 253-255

ISSN : 1774-4296

\section{Référence électronique}

Geneviève Hoffmann, «David Bouvier et Danielle van mal-maeder (éd.), Tradition classique : dialogues avec l'Antiquité », Anabases [En ligne], 14 | 2011, mis en ligne le 01 octobre 2011, consulté le 22 septembre 2020. URL : http://journals.openedition.org/anabases/2357 ; DOI : https://doi.org/10.4000/anabases. 2357

Ce document a été généré automatiquement le 22 septembre 2020

(c) Anabases 


\title{
David BOUVIER et Danielle VAN MAL- MAEDER (éd.), Tradition classique : dialogues avec l'Antiquité
}

\author{
Geneviève Hoffmann
}

\section{RÉFÉRENCE}

David BOUVIER et Danielle VAN MAL-MAEDER (éd.), Tradition classique : dialogues avec

l'Antiquité, Revue Études de Lettres, Lausanne, Université de Lausanne, 2010, 294 p.

26 francs suisses / ISBN 978-2-940331-22-2.

1 Si les deux premières contributions de ce volume sont des conférences inaugurales, en l'occurrence celles données par les auteurs responsables de la publication, les treize autres sont les communications présentées en novembre 2006 à l'université de Lausanne lors du colloque qui s'est tenu à l'ouverture de la discipline «Tradition classique » à la faculté des lettres, pour faire émerger, entre des chercheurs de la même institution, un questionnement et un savoir communs sur l'histoire de la transmission et de la réception du patrimoine antique.

2 Chaque étude est précédée par un résumé en français et complétée par une bibliographie plus ou moins développée. L'avant-propos annonce d'emblée le caractère incomplet et peu systématique du volume. D'Homère à la bande dessinée, ce sont en effet des sources bien différentes qui sont interrogées dans leur rapport à l'Antiquité. En l'absence de conclusion et d'index, il revient au lecteur de tisser les liens entre les enquêtes proposées et de trouver une cohérence à un ensemble hétérogène.

Dans sa présentation des «Lieux et non-lieux de Troie» (p.9-38), David Bouvier s'interroge sur le succès médiatique du film de Wolfgang Petersen, Troy, présenté hors compétition au festival de Cannes de 2004. Après avoir retracé l'histoire de cette «vieille énigme » que fut le site de Troie depuis les fouilles de Schliemann et relevé comment Rome et les dynasties européennes ont su utiliser la référence aux Troyens, il 
souligne, en cette aube du XxI $\mathrm{e}^{\mathrm{e}}$ siècle, la convergence idéologique entre les intérêts de l'archéologie et les autorités d'Ankara, désireuses d'être rattachées à la culture occidentale. Dès Mehmet II, présent sur le site de Troie neuf ans après la prise de Constantinople, les Turcs ont voulu s'inventer un héros fondateur, Turcus, venu de Troie tout comme Énée. Pour David Bouvier, le scénario de Petersen, très favorable aux Troyens, a permis à Hollywood « de faire un clin d'œil à la Turquie », base de départ des raids contre l'Irak. La source de la contribution de Danielle van Mal-Maeder : « La Peste, les Dieux et les Hommes. Cheminements d'une tradition» (p.39-59) est un poème bucolique du Iv siècle du rhéteur Severus Sanctus Endelechius : De la mort des boeufs, où elle retrouve la trace des Bucoliques et des Géorgiques de Virgile, que la littérature chrétienne s'est approprié. Puis de Lucrèce, elle remonte à Thucydide et à ses pages sur le typhus qui sévit à Athènes en 430. L'auteur conclut son cheminement par La Peste de Camus, lui-même lecteur de Lucrèce et de Thucydide, pour affirmer «le principe de pérennité de la culture antique»(p.55). C'est à l'histoire de la traduction que s'intéresse Etienne Barilier. À partir d'un mot de l'Antigone de Sophocle: deinon (v. 332-333) et de ses interprétations, il pose la question : «L'Homme est-il merveilleux ou terrible? » (p. 61-79). Depuis la Renaissance jusqu'à Lacan en passant par Hölderlin et Heidegger, la pluralité des sens donnés à cet adjectif, quasiment intraduisible, renvoie selon lui à l'image que l'Européen se donne de lui-même, entre l'émerveillement devant sa propre histoire et la terreur de l'abîme. En éclairant la conversion de Paul sur le chemin de Damas par les théories actuelles sur la créativité, Pierre-Yves Brandt (p. 81-98) souligne que l'action qui fit de Paul un possédé résultait, tout comme une découverte scientifique, d'une longue préparation psychologique que seul l'enjeu théologique a conduit à présenter comme un appel et une révélation. Francesco Gregorio et Catherine König-Pralong retracent l'histoire de «l'autoportrait du philosophe : du Lycée grec à l'Université médiévale » (p. 99-116). Si l'éloge que fait Aristote de la vie philosophique dans l'Ethique à Nicomaque, fonde leur analyse, leur enquête se concentre sur la traduction donnée par l'évêque Robert Grosseteste en 1247 et sur le commentaire établi par dominicain Albert le Grand en 1250. À une forme de vie qui fait du philosophe dans le système aristotélicien l'unique bienheureux sur terre, l'interprétation d'Albert le Grand, influencée par Avicenne et Averroès, ajoute la possibilité d'« intelliger les choses divines » (p. 113), pour entrer dans l'universel et le céleste. Ainsi émerge dans la pensée médiévale la figure du philosophe divin. Gabriella Aragione s'intéresse à l'accusation de plagiat comme arme idéologique entre écoles concurrentes et auteurs chrétiens des $\mathrm{II}^{\mathrm{e}}$ et $\mathrm{III}^{\mathrm{e}}$ siècles (p. 117-138). Si la dénonciation du plagiaire traduit une volonté d'effacement de la mémoire, la notion de tradition valorise au contraire la transmission d'un savoir. «Mais qui était Hypathie? " ainsi débute l'analyse proposée par Anne-Françoise Jaccottet (p. 139-158) sur cette excellente philosophe et mathématicienne qui donnait des conférences à Alexandrie au IV siècle de notre ère. Sa mort atroce en 415 en fit une victime de l'hystérie chrétienne et sa figure tour à tour réinventée et réutilisée au fil des siècles l'imposa dans les luttes contre l'obscurantisme à l'époque des Lumières comme dans celles des féministes du $\mathrm{xx}^{\mathrm{e}}$ siècle, sans oublier son apparition sous les traits de Rachel Weisz dans le film Agora (2009). Marco Praloran (p. 159-172) a retenu «le monologue lyrique et la narration épique " comme témoins de la réception des Classiques dans la littérature de la Renaissance italienne. Il consacre le premier volet de son étude à Pétrarque, le second au genre chevaleresque, illustré par Matteo Maria Boiardo. Alors que le poète a opéré la synthèse du stoïcisme et de la perspective chrétienne, l'épopée italienne, dégagée de 
toute influence classique, a pourtant récupéré l'allitération, figure de style caractéristique de l'épopée antique. Neil Forsyth rappelle que John Milton fut poète et révolutionnaire (p. 173-187). Son œuvre fait de multiples références à la poésie grécolatine, mais elle se nourrit aussi de la littérature dite « républicaine » qui occupait une place de choix dans la rhétorique de la Révolution anglaise du XVII siècle. Relire Athalie, Tragédie tirée de l'écriture sainte à la lumière des sources hébraïques et grecques sous la conduite d'Adrien Paschoud (p.189-203) permet de comprendre "la lutte des sacralités " qui se joue dans les discours et la dynamique de la pièce. Le paganisme antique, présenté comme "un véritable embrayeur », sert de contrepoint au message biblique tout en exerçant un pouvoir de fascination. Christian Michel s'intéresse au «basculement de la tradition classique » dans le domaine artistique à partir des années 1750 (p. 205-216), basculement qui s'opéra grâce à J. Winckelmann et qui inaugura un Retour à l'antique selon des modalités différentes de celles de la Renaissance. Dans son analyse qui a pour titre, "Rodolphe Töpffer et l'Antiquité»(p.217-228), Daniel Maggetti retrace l'histoire des rapports que le célèbre Genevois a entretenus avec les études classiques. La connaissance du latin et du grec l'a promu socialement et a nourri son inspiration. Toutefois son accession au professorat en 1832 a marqué la fin de son insolence salutaire. Par la comparaison entre les héros des deux BD antiquisantes les plus célèbres (p. 229-249), Alix l'intrépide, né en 1948, et Astérix le Gaulois, né en 1959, Alain Corbellari cherche à cerner le message qu'ils délivrent sur la France de la Cinquième République. Si la première BD se veut bien documentée et la seconde plus désinvolte quant à la précision historique, l'une et l'autre savent instrumentaliser le rapport à l'Antiquité pour défendre le mythe d'une société multiculturelle ou pour dénoncer la pax americana. Par la reprise d'un titre de Roland Barthes : "Comment représenter l'antique? ", Pierre Voelke invite le lecteur à l'étude des mises en scène des tragédies de Sophocle, de l'Antigone de l'Odéon (1844) aux spectacles d'Antoine Vitez $(1966,1971,1986)$ en passant par l'Orestie de Jean-Louis Barrault et les Troyennes de Sartre (p. 251-273). Qu'elle recherche la reconstitution ou l'actualisation, la représentation du drame antique doit toujours préserver la puissance du verbe tragique. Le volume se termine par la contribution de Rudolf Wachter (p. 275-292): «Fouiller les mots » comme le ferait un archéologue pour inscrire la tradition classique dans l'étymologie et les variantes de sens au fil du temps, "car notre langue est le miroir le plus clair de notre culture et de sa genèse » (p. 275).

Ce volume en témoigne s'il en était besoin : l'Antiquité fut bien un répertoire de mots, de formes et d'idées dans lequel chaque époque a puisé pour les instrumentaliser. Le spécialiste de l'Antiquité, philologue, historien ou archéologue, doit-il être « un briseur de rêves " pour rétablir la complexité et l'authenticité de l'original derrière le miroir déformant et toujours simplificateur de la réception? Est-ce là sa vocation et son devoir? Seule Anne-Françoise Jaccottet pose cette question qui est loin d'être secondaire. Il est certain que l'attention portée à la tradition classique et à sa réception est révélatrice d'une interrogation quant au devenir des Humanités. Ce patrimoine culturel qui dans le passé a servi à exprimer une identité, un imaginaire et une distinction, est aujourd'hui menacé. À ce titre - et ce recueil en témoigne, les dialogues avec l'Antiquité pourraient être un recours pour préserver la mémoire de la tradition classique. 


\section{AUTEURS}

\section{GENEVIĖVE HOFFMANN}

Université de Picardie Jules Verne

genevieve.hoffmann@wanadoo.fr 